[pp. 93-111]

Vol. 4(2), Julio - Diciembre, 2016

http://dx.doi.org/10.15359/rnh.4-2.5

\title{
Seguridad alimentaria y
}

nutricional: su fomento

\section{desde el aula de inglés de "la universidad necesaria"}

Food and Nutritional Security: Promoting It from the Classroom of English at the "Necessary University"

David Enoc Villalobos Betancourt

Costa Rica

david.villalobos.betancourt@una.cr

\section{Resumen}

Este artículo hace un breve recorrido por las bases teóricas que sustentan el concepto de seguridad alimentaria y nutricional (SAN), algunas de las causas de la inseguridad alimentaria y la necesidad de la toma de decisiones acertadas. Se recurre a los fundamentos de la Universidad Nacional, como "la universidad necesaria" para crear conciencia, desde el contexto de las aulas, sobre la seguridad alimentaria y nutricional de nuestras comunidades. Se ejemplifica, desde el contexto de las clases de inglés como lengua extranjera, cómo se puede motivar a la discusión y al debate para una mejor visión de la alimentación con base en actividades que no solo contribuyen al enriquecimiento del inglés, su vocabulario y sintaxis, sino que motiva a los estudiantes a tomar conciencia sobre sus necesidades nutricionales y sus derechos. Se concluye que Costa Rica no

ha logrado cumplir la Meta de la Cumbre Mundial sobre la Alimentación de 1990-1992 a 2014-2016, ya que todavía reporta más de 200000 personas en ese estado. Además, se indica hace énfasis en el papel que desempeñan los docentes universitarios en 
el aprendizaje para que las nuevas generaciones tomen conciencia y elijan las mejores decisiones sobre el tema.

Palabras clave: seguridad alimentaria y nutricional; nutrición; educación para la salud; enseñanza del inglés; universidad.

\begin{abstract}
This article briefly reviews theoretical foundations underlying the concept of food and nutritional security (SAN, for its acronym in Spanish), some of the causes of food insecurity, and the need for right decision-making. It has recourse to the foundations of the National University as "the necessary university" to raise awareness, from the context of the classroom, on food and nutritional security of our communities. From the context of teaching English as a foreign language, it is shown how to promote the discussion and debate for a better view on food, based on activities that not only contribute to the enrichment of the English language, its vocabulary, and syntax, but that encourage students to become aware of their nutritional needs and rights. It is concluded that Costa Rica has not been able to fulfill the goal of the World Food Summit from 1990-1992 to 2014-2016, because more than 200,000 people are still reported in state of malnutrition. In addition, emphasis is placed on the role of university teachers in the learning for the new generations to be aware and make the best decisions on the subject.
\end{abstract}

Keywords: Food and nutritional security; nutrition; health education; teaching English; university.

\title{
Introducción
}

Al estudiar el Mapa del hambre de formulado por la Organización de las Naciones Unidas para la Alimentación y la Agricultura (FAO 2015b), se nota claramente que Costa Rica aparece en el grupo de los países que tiene un porcentaje muy bajo ( $5 \%$ o menos) en cuanto a la prevalencia de la subalimentación de la población para el período 2012-2014 y de igual forma aparece dentro de la clasificación de las regiones que alcanzaron el primer objetivo de desarrollo del milenio meta 1.C, el cual se puede leer textualmente en el mismo documento "reducir a la mitad, entre 1990-2092 y 2015, la proporción de personas subalimentadas, o reducir esta proporción a menos del $5 \%$ ". Esto, en palabras de Léon, Martínez, Espíndola y Schejtman (2004), muestra “...en general un bajo grado de vulnerabilidad" (p. 14). Sin embargo, en cuanto a la consecución de la meta de la Cumbre Mundial sobre la Alimentación de 1990-1992 a 2014-2016, Costa Rica 
claramente aparece dentro del grupo de países que tienen una "Meta no alcanzada, sin progreso, o en proceso de deterioro" (FAO, 2015b).

Con base en el cuadro 1 del Panorama de Seguridad Alimentaria y Nutricional en Centroamérica y República Dominicana 2014 (p. 4), se puede determinar que para el período 1990-1992, Costa Rica reportaba 164000 personas subalimentadas. El período 2000-2002 reporta 205000 personas, en el 2005-2007 el número se incrementa a 246000 , ya para el 2008-2010 esta cifra alcanza las 244000 y este número se incrementa a 287000 personas para la evaluación del período 2012-2014. Esto demuestra un empeoramiento en las cifras. Muy a pesar de esto, León, Martínez et al. (2004) aseguran que "Costa Rica muestra un suministro estable y superior en alrededor de $30 \%$, aunque ello no sería suficiente para erradicar la subnutrición, no obstante, su bajo nivel de desigualdad de acceso a los alimentos" (p. 12). A esto se ha de agregar el hecho de que para FAO (2014):

...la mayoría de los países de la región enfrentan 'una doble carga de malnutrición' y han visto incrementarse sus cifras de sobrepeso y obesidad hasta el punto [sic] que más del $40 \%$ de los hombres y el $50 \%$ de las mujeres adultas en todos los países sufren sobrepeso. (p. XIII)

\section{La malnutrición}

Los problemas de la 'malnutrición' trascienden fronteras y afectan a muchos países. Sin embargo, los de la subalimentación afectan especialmente al continente africano, al asiático, y al americano. Gustavo Gordillo en su entrevista con Luisa Goncalves (Pérez, 2012), establece que "La cruda realidad es que hoy casi 840 millones de personas en todo el mundo padecen el problema del hambre. Pero, al mismo tiempo, los alimentos que se producen a nivel mundial alcanzan para todos los habitantes de la tierra" (p. 225). Esto ciertamente es una paradoja, pero es el panorama que predomina en un mundo plagado por las desigualdades. Gordillo con Luisa Goncalves (en Pérez, 2012) indica que hay "necesidad de una mayor producción de alimentos, facilitar el acceso de todos a los alimentos, asegurar que los suministros de alimentos lleguen establemente a las personas y la necesaria inocuidad de los alimentos" (p. 231).

En ese sentido es importante, entonces, poder garantizar que los alimentos estén disponibles para todas las personas y para esto se ha llegado a acuerdos internacionales, como, por ejemplo, La Cumbre Mundial Sobre Alimentación de 1996, 
que marcan las pautas para la consecución de los objetivos propuestos y garantizar así la seguridad alimentaria y nutricional.

La FAO (2016) es clara al establecer:

La seguridad alimentaria se da cuando todas las personas tienen acceso físico social y económico permanente a alimentos seguros, nutritivos y en cantidad suficiente para satisfacer sus requerimientos nutricionales y preferencias alimentarias, y así poder llevar una vida activa y saludable. (párr. 1)

Desafortunadamente, esto no es la realidad mundial y esto lo evidencian las cifras de hambre que todavía imperan. Es un horizonte duro e incierto; desnutrición y hambre van de la mano, pero hay otros factores que entran en juego, como lo propone Schejtman (2005), quien indica que “...la desnutrición aparece como resultado inmediato del hambre y/o de insuficiencias en materia de salud, educación y equipamiento del hogar... y de la forma en que los alimentos se distribuyan al interior de la familia" (énfasis agregado) (p. 25). Nótese que la falta de educación forma parte de las causas de la desnutrición, ya que es por medio de esta que las personas pueden hacer mejores elecciones en cuanto a lo que comen para una vida más saludable. La educación nutricional es de suma importancia y esta se puede definir así:

...a grandes rasgos como una serie de programas integrales que incluyen un conjunto de intervenciones en materia de información dirigidas a aumentar los conocimientos de los consumidores sobre lo que constituye una buena nutrición. El objetivo final es un cambio en el comportamiento para que las personas elijan dietas más nutritivas y estilos de vida más sanos. (FAO, 2015a p. 77)

La educación nutricional es, entonces, una herramienta de gran valor en el logro de la seguridad alimentaria y nutricional para todas las personas.

En cuanto a las seguridad alimentaria y nutricional (SAN en adelante), Costa Rica ha establecido un plan nacional y este ha sido puesto en práctica (Plan Nacional de Seguridad Alimentaria y Nutricional 2011-2015). En el documento propuesto se ve la educación reflejada en las políticas. En la política 2, se establece la "Promoción de hábitos y estilos de vida saludables que permitan mejorar la salud y nutrición de la población considerando los lineamientos recomendados a nivel nacional e internacional" (Ministerio de Salud, 2011, p. 26). Y el inciso 2.1 de 
esa misma política, establece el "Fortalecimiento de la información, educación y comunicación a los consumidores sobre la calidad y la importancia del consumo de productos inocuos y saludables, en coordinación con la industria alimentaria..." (Ídem, p. 26). Pero no podemos esperar que solo el Ministerio de Salud sea el responsable de esta tarea.

Los centros educativos, desde sus etapas iniciales con la niñez, deben procurar la mejor educación para un desarrollo de buenas elecciones dietéticas y el desarrollo de hábitos alimenticios saludables, especialmente en este momento histórico cuando los alimentos procesados, poco a poco, han ido desplazando de las mesas de muchos hogares costarricenses los alimentos íntegros que en otros tiempos eran la elección número uno de nuestro pueblo. Y si en la escuela se puede lograr mucho en cuanto a seguridad alimentaria y nutricional se refiere, cuanto más en el nivel universitario, cuando se ha alcanzado una mejor capacidad de análisis y el individuo es competente para la toma de decisiones. Es aquí donde las aulas de la Universidad Nacional, la universidad necesaria, se convierten en verdaderas oportunidades de cambio.

\section{La Universidad Nacional y la seguridad alimentaria y nutricional}

La Universidad Nacional desempeña un papel preponderante como agente de cambio, en la lucha por diversas causas y entre ellas es de suma importancia considerar la SAN. En el contexto de la Declaratoria de la UNA por la Madre Tierra y tomando en cuenta los cuatro componentes de la SAN mencionados por Gustavo Gordillo en su entrevista con Luisa Goncalves (Pérez, 2012), los cuales incluyen la producción de alimentos; el acceso a ellos; la estabilidad de los suministros de estos; y la inocuidad de los alimentos, los cuales deben ser saludables y nutritivos, se observa que hay relación íntima entre la defensa de la tierra y la SAN, puesto que del valor y la seguridad de la tierra depende la producción de alimentos, así como su valor energético y su inocuidad.

La UNA, como "La Universidad necesaria" y mediante diversas gestiones que conlleven al cambio y al crecimiento no solo académico y de la comunidad, sino también humano, puede contribuir por medio de acciones educativas hacia una sociedad con un porcentaje de malnutrición más reducido o nulo. En palabras del exrector Dr. Olman Segura mencionado por Núñez (2008), en el prólogo del libro Hacia la universidad necesaria: "La UNA ahora como antes, continuará trabajando en acciones integrales que direccionen a la sociedad hacia planes superiores de 
bienestar, equidad, sostenibilidad y libertad democrática, mediante paradigmas que permitan transformar y revalorar el desarrollo humano" (p. 12). La SAN es evidentemente fundamental para que haya bienestar, equidad, y sostenibilidad. No se puede pensar en desarrollo pleno del individuo como ser humano, si carece de los suministros alimenticios necesarios o si estos son perjudiciales o carentes de las propiedades nutricionales y el valor energético necesario para desempeñarse a capacidad total.

Es aquí donde la función del personal docente como agente de cambio hacia la mejora de nuestras comunidades estudiantiles toma fuerza, ya que es este quien pasa la mayor parte del tiempo en las aulas y en las actividades pedagógicas complementarias, con el estudiantado. Tomo, además, como fundamentos dos propuestas de la Declaratoria Institucional 2016: Año de la UNA por la Madre Tierra, las cuales marcan la pauta hacia (1) la implementación de:

...prácticas científicas y de la vida cotidiana que favorezcan el equilibrio de los ecosistemas y... (la promoción de) ... una ética del cuidado personal y colectivo, como un compromiso impostergable para enfrentar la situación crítica que vive la humanidad y el planeta. (Declaratoria Institucional 2016, párr. 4)

Y (2) la promoción de "...una actividad práctica de la vida universitaria sustentada en una sensibilidad de derechos humanos que fortalezca el humanismo, la solidaridad social, la equidad, la libertad y el respeto a la dignidad humana" (Declaratoria Institucional 2016, párr. 4).

Como docentes y en nuestro trabajo en las aulas estamos en una posición de poder ver estos ideales de "la ética de cuido personal", "derechos humanos", "solidaridad social", "equidad" y "el respeto por la dignidad humana" consolidados en la SAN, de la cual todos debemos ser partícipes.

\section{La SAN desde el contexto del aula}

\section{Población}

Este trabajo se llevó a cabo con estudiantes de primer ingreso de la carrera de Diplomado en Inglés de la Universidad Nacional, Campus Liberia, durante el I ciclo del 2016. El grupo estaba compuesto por una población de 22 estudiantes (13 mujeres y 9 hombres), cuyas edades oscilan entre los 17 y 24 años. 


\section{Metodología de estudio}

Para el logro de los objetivos de esta propuesta, se vincularon los objetivos de estudio del curso, propiamente la unidad 3 del libro de clase, Interactions I, Integrated Skills Edition, Capítulo 3, "Living to Eat or Eating to Live?" (¿Vivir para comer o comer para vivir?). En este capítulo la temática se orienta hacia hábitos alimenticios y de compra para lograr los objetivos. Las actividades de clase se distribuyeron a lo largo de cuatro sesiones durante las cuales se discutió el vocabulario, las estructuras gramaticales, y las frases fórmulas necesarias, entre ellas las preguntas "What is your height?, What is your weight?, y What is your body mass index?". Otras preguntas incluyeron: "What do you usually eat?, What did you eat yesterday?, What did you eat toda y?"

En conjunto con la preparación necesaria para la activación de conocimientos previos y para lograr el dominio de los nuevos contenidos, se condujeron prácticas de clase. Por ejemplo, el repaso y reconocimiento del vocabulario más común de cereales (bread, rice, wheat, spaghetti, pasta, cereal...), frutas y vegetales (orange, pineapple, bananas, cabbage, beets, spinach, etc.), meats (chicken, beef, pork, fish...) productos lácteos (dairy, milk, cheese, sour cream...), azúcar y grasas (sugar, fat, oil, olive oil, candy...). Estos alimentos se contextualizaron en la Pirámide alimenticia del Departamento de Agricultura de los Estados Unidos (USDA por sus siglas en inglés), la cual está disponible en línea y es de dominio público, por lo que no se necesita de un permiso para reproducirlo y compartirlo con los estudiantes. De igual manera, se tomó en cuenta la imagen "The Healthy Eating Plate" la cual está disponible en línea y cuenta con permisos para usarse para propósitos educativos (ver anexos 1 y 2). En conjunto con estos instrumentos, se discutieron, a grandes rasgos, las cantidades necesarias medidas en porciones, de alimentos que cada quien debe consumir diariamente. Una vez que se logró el dominio de los objetivos propuestos, se procedió a hacer un primer ejercicio, de forma individual, con base en la pirámide alimenticia, el cual consistió en una hoja de trabajo, para cada estudiante, con cuatro ilustraciones de pirámides alimenticias con solo pequeños cuadros equivalentes al número de porciones que cada persona debe comer (ver anexo 3). Por medio de la técnica de lluvia de ideas, se le ayudó al estudiantado a completar la pirámide 1 y 2 con base en lo que había comido el día anterior y en el transcurso del día actual. Para hacer esto, por ejemplo, la pirámide presenta en el segmento de frutas 4 cuadritos, cada uno corresponde a una porción de fruta. Si el estudiante había comido 4 
porciones de frutas, coloreaba todos cuadritos y así sucesivamente con todos los demás aspectos. Una vez que se habían completado los días 1 y 2 , se le asignó a cada estudiante continuar con la dinámica en el hogar de forma individual por dos días más hasta completar las cuatro pirámides. Al finalizar, el recuento de los cuatro días, se les instruyó para tabular los datos y preparar un informe oral. Este debía incluir un autoanálisis de las acciones acertadas y desacertadas que cada quien había realizado y qué hacer para mejorar los hábitos alimenticios y la malnutrición. Por medio de este ejercicio, gran cantidad de estudiantes pudieron ver cómo sus dietas incluían una cantidad reducida de frutas y vegetales contra un exceso de cereales, grasas o azúcares.

Para darles continuidad a las actividades de la temática, se trajo una pesa y una cinta métrica al aula y se instruyó al grupo a cooperar para pesarse y medirse entre unas personas y otras. Luego se les pidió responder a las preguntas: What is your height? y How much do you weigh? Con base en el peso y la talla, se hizo el cálculo del índice de masa corporal (IMC) y, con esta información, se determinó si tienen un peso adecuado o no con base en los rangos provistos por la OMS (2015) y así se respondió a la pregunta, What is your Body Mass Index?

Posteriormente y para determinar la ingesta de alimentos, se utilizó la técnica del Recordatorio de 24 horas, por medio de un instrumento elaborado en lengua inglesa. Para este propósito se le entregó a cada estudiante una encuesta, la cual puede ser vista en los anexos, y en esta se les solicitó anotar todos los alimentos consumidos en las últimas 24 horas, en el desayuno, almuerzo, cena, además de los entremeses consumidos. Esto se hizo por medio de una actividad en conjunto. El encabezado debió ser completado con la observación obtenida por medio de la toma de peso, la medida de estatura y el cálculo del IMC, además de información básica del estudiante como el género y la edad. Para ayudarles a recordar, se les solicitó estimar el tiempo en que consumieron los alimentos, el lugar dónde los consumieron y las cantidades estimadas en medidas caseras y en gramos. Seguidamente se tabularon todos los datos. Se compartieron los resultados y se les motivó a discutir mejoras en la alimentación para establecer mejoras en la SAN. Ejemplos de frases utilizadas incluyeron: "I am underweight. I am overweight. I am obese. I have a normal weight. I need to gain weight. I need to lose weight. I must eat more vegetables". 


\section{Resultados}

En el análisis de la información obtenida se diseñaron las siguientes tablas para realizar el análisis de la información de manera más efectiva. En la tabla 1, correspondiente al grupo de las mujeres, se puede observar que seis de ellas presentan un peso normal, seis preobesidad y una peso insuficiente.

\section{Tabla 1}

Organización de los datos de la población femenina

\begin{tabular}{lllllll}
\hline & Género & Edad & Talla & Peso & IMC & Resultado \\
\hline 1. & F & 17 & 146 & 46,0 & 21,6 & Normal \\
2. & F & 17 & 155 & 49,0 & 20,4 & Normal \\
3. & F & 17 & 170 & 60,0 & 20,8 & Normal \\
4. & F & 18 & 156 & 46,0 & 18,9 & Normal \\
5. & F & 24 & 170 & 59,0 & 20,4 & Normal \\
6. & F & 18 & 163 & 63,0 & 23,7 & Normal \\
7. & F & 17 & 166 & 69,0 & 25,0 & Preobesidad \\
8. & F & 19 & 172 & 74,0 & 25,0 & Preobesidad \\
9. & F & 21 & 156 & 72,0 & 29,6 & Preobesidad \\
10. & F & 17 & 166 & 71,0 & 25,8 & Preobesidad \\
11. & F & 18 & 167 & 80,0 & 28,7 & Preobesidad \\
12. & F & 18 & 153 & 63,0 & 26,9 & Preobesidad \\
13. & F & 19 & 163 & 44,5 & 16,7 & Peso insuficiente \\
\hline
\end{tabular}

Nota: Elaboración propia, 2016.

Al establecer cálculos porcentuales de la muestra, se observa que un $46 \%$ presenta un peso normal. Otro $46 \%$ presenta preobesidad y solamente un $8 \%$ presentan peso insuficiente.

En lo que se refiere a la población masculina, los datos se organizaron en la tabla 2. 


\section{Tabla 2}

Análisis de los datos de la población masculina

\begin{tabular}{lllllll}
\hline & Género & Edad & Talla & Peso & IMC & Resultado \\
\hline 1. & M & 17 & 1,77 & 64,5 & 20,6 & Normal \\
2. & M & 17 & 1,72 & 60,0 & 20,3 & Normal \\
3. & M & 18 & 1,83 & 73,0 & 21,8 & Normal \\
4. & M & 19 & 1,68 & 70,0 & 24,8 & Normal \\
5. & M & 19 & 1,66 & 57,5 & 20,9 & Normal \\
6. & M & 20 & 1,69 & 80,0 & 28,0 & Preobesidad \\
7. & M & 17 & 1,77 & 118 & 37,7 & Obesidad de tipo II \\
8. & M & 17 & 1,69 & 53,0 & 18,6 & Peso insuficiente \\
9. & M & 17 & 1,71 & 53,0 & 18,1 & Peso insuficiente \\
\hline
\end{tabular}

Nota: Elaboración propia, 2016.

Poniendo esta información en términos de porcentajes, se puede observar que entre los varones, cinco de ellos presentan un peso normal (56\%), uno tiene preobesidad (11\%), uno presenta obesidad grado I (11\%) y dos varones tienen peso insuficiente $(22 \%)$.

Al analizar el total de la muestra completa, 11 personas tienen un peso normal. Siete personas presentan preobesidad; una persona tiene obesidad de tipo II y tres personas tienen peso insuficiente. Se puede establecer que solamente $50 \%$ del total tiene un peso normal, mientras que el $32 \%$ tienen preobesidad, el $4 \%$ muestra obesidad tipo II y $14 \%$ presentan peso insuficiente. En términos de malnutrición, podemos hablar de un $50 \%$ de la población con un peso normal frente a un $50 \%$ en estado de "malnutrición.

La información recolectada indica que el mayor consumo de alimentos durante el desayuno incluye el pan, la fruta, el queso y el café, seguidos de jugo y gallo pinto.

Los alimentos de mayor consumo durante el almuerzo incluyen el arroz, la carne, la ensalada, los vegetales y los frijoles. Para la cena se observa que hay una tendencia a comer mayormente arroz, ensalada y carne, además de frijoles y pan. Como entremés se consumen más las galletas, el jugo, la fruta y los pasteles. 


\section{Conclusiones}

Costa Rica, muy a pesar de su bajo porcentaje de desnutrición, no ha logrado cumplir la "Meta Relativa al Hambre" de la Cumbre Mundial sobre la Alimentación de 1990-1992 a 2014-2016, ya que todavía reporta más de 200000 personas en ese estado. De esta manera, todavía quedan muchos esfuerzos por hacer para lograr los objetivos propuestos.

En cuanto a la SAN se refiere, el tema de la educación es de suma importancia y el personal docente universitario desempeña un papel muy importante para promover situaciones de aprendizaje que ayuen a tomar consciencia a las nuevas generaciones en la toma de decisiones.

Al analizar los datos provistos por la población encuestada, se puede concluir que solo un $50 \%$ de ellos presentan un peso normal. El otro $50 \%$ presenta, peso insuficiente, preobesidad u obesidad, lo cual evidencia un alto porcentaje de malnutrición. En esta pequeña muestra, entonces, se ven reflejados los altos índices de malnutrición que se reportan el Panorama de la Seguridad Alimentaria y Nutricional de Centroamérica y República Dominicana 2014, ya que el $32 \%$ tiene preobesidad y el $4 \%$ muestra obesidad tipo II. Esto en términos generales, representa un $50 \%$ de la población que se encuentra en estado de "malnutrición".

En cuanto a los hábitos alimenticios, es claro que hay una preferencia por el consumo mayormente de carbohidratos como el pan y el arroz, seguido de alimentos proteínicos como los frijoles y la carne.

Se ve, sin embargo, que la población estudiada en términos generales también consume frutas y vegetales, pero probablemente las cantidades consumidas no sean las adecuadas.

Se evidenció que la información recopilada motivó al estudiantado a pensar en sus hábitos alimenticios, ya que la dinámica de clase no solo le permitió desarrollar competencia en inglés en el tema de la alimentación, sino que también les permitió pensar reflexionar sobre los cambios que deben realizar para una mejor nutrición. El hecho de vincular contenidos de clase con la SAN, fue de mucho provecho y generó mucho interés e interacción. 


\section{Recomendaciones}

- Establecer propuestas desde la academia y en el contexto del aula universitaria que guíen al estudiantado a tomar conciencia de la SAN en el mundo, pero especialmente en el país y en el seno de sus hogares.

- Involucrar al estudiantado en diversas actividades lúdicas y académicas que le motiven a desarrollar hábitos alimenticios que incluyan solamente comestibles seguros e inocuos.

- Motivar al estudiantado que presente una situación de malnutrición a consultar con profesionales de la salud competentes.

- Continuar con el estudio de la población, en colaboración con profesionales de la salud, para afinar conclusiones con respecto a las causas de sobrepeso, pero también del porcentaje de peso insuficiente que existe en el grupo para poder articular planes que motiven al cambio y a la reducción de las cifras de malnutrición imperantes en la comunidad.

\section{Referencias}

Organización de las Naciones Unidas para la Alimentación y la Agricultura (FAO). (2014). Panorama de la Seguridad Alimentaria y Nutricional de Centroamérica y República Dominicana 2014. Ciudad de Panamá. Recuperado de: www.fao.org/3/a-i4349s.pdf

Organización de las Naciones Unidas para la Alimentación y la Agricultura (FAO). (2015a). El estado mundial de la agricultura y la alimentación; la protección social y la agricultura: Romper el ciclo de la pobreza rural. Recuperado de: www.fao.org/3/a-i4910s.pdf

Organización de las Naciones Unidas para la Alimentación y la Agricultura (FAO). (2015b). Mapa del hambre. Recuperado de: www.fao.org/3/a-i4674s.pdf

Organización de las Naciones Unidas para la Alimentación y la Agricultura (FAO). (2016). Estadísticas sobre seguridad alimentaria. Recuperado de: http://www. fao.org/economic/ess/ess-fs/es/

Harvard University. (2016). Healthy Eating Plate. [imagen]. Recuperado de: https://www.hsph.harvard.edu/nutritionsource/healthy-eating-plate/ 
León, A., Martínez, R., Espíndoloa, E. y Schejtman, A. (2004). Pobreza, hambre y seguridad alimentaria en Centroamérica y Panamá. Santiago de Chile. Naciones Unidas. Recuperado de: http://repositorio.cepal.org/ handle/11362/6077?locale-attribute $=\mathrm{es}$

Ministerio de Salud. (2011). Plan Nacional de Seguridad Alimentaria y Nutricional 2011-2015. San José, Costa Rica. Recuperado de: https://www.ministeriodesalud.go.cr/index.php/biblioteca-de-archivos/sobre-el-ministerio/politcas-y-planes-en-salud/planes-en-salud/1335-plan-nacional-de-seguridad-alimentaria-y-nutricional-2011-2015/file

Núnez, B. (2008). Hacia la Universidad necesaria. Heredia, Costa Rica: EUNA.

Organización de las Naciones Unidas (ONU). (1996). Informe de la Cumbre Mundial sobre la Alimentación 13 - 17 de noviembre de 1996. Recuperado de: http://www.fao.org/docrep/003/w3548s/w3548s00.htm

Organización Mundial de la Salud (OMS). (2015). Obesidad y sobrepeso. Recuperado de: http://www.who.int/mediacentre/factsheets/fs311/es/

Pérez E, L. (2012). Diálogo con Gustavo Gordillo sobre Ambiente y seguridad alimentaria. Revista Perspectivas Rurales Nueva Época, 0(10). Recuperado de: http://www.revistas.una.ac.cr/index.php/perspectivasrurales/article/ view/3578

Schejtman, A. (2005). Elementos para una estrategia integral de seguridad alimentaria en Centro América. Anuario de Estudios Centroamericanos, 31, 7-47. Recuperado de: http://www.jstor.org/stable/25661384

Universidad Nacional (UNA). (2016). Declaratoria Institucional 2016: Año de la UNA por la Tierra. Recuperado de: http://www.madretierra.una.ac.cr/ 


\section{Anexos}

1. Food Pyramid Guide (Imagen de dominio público).

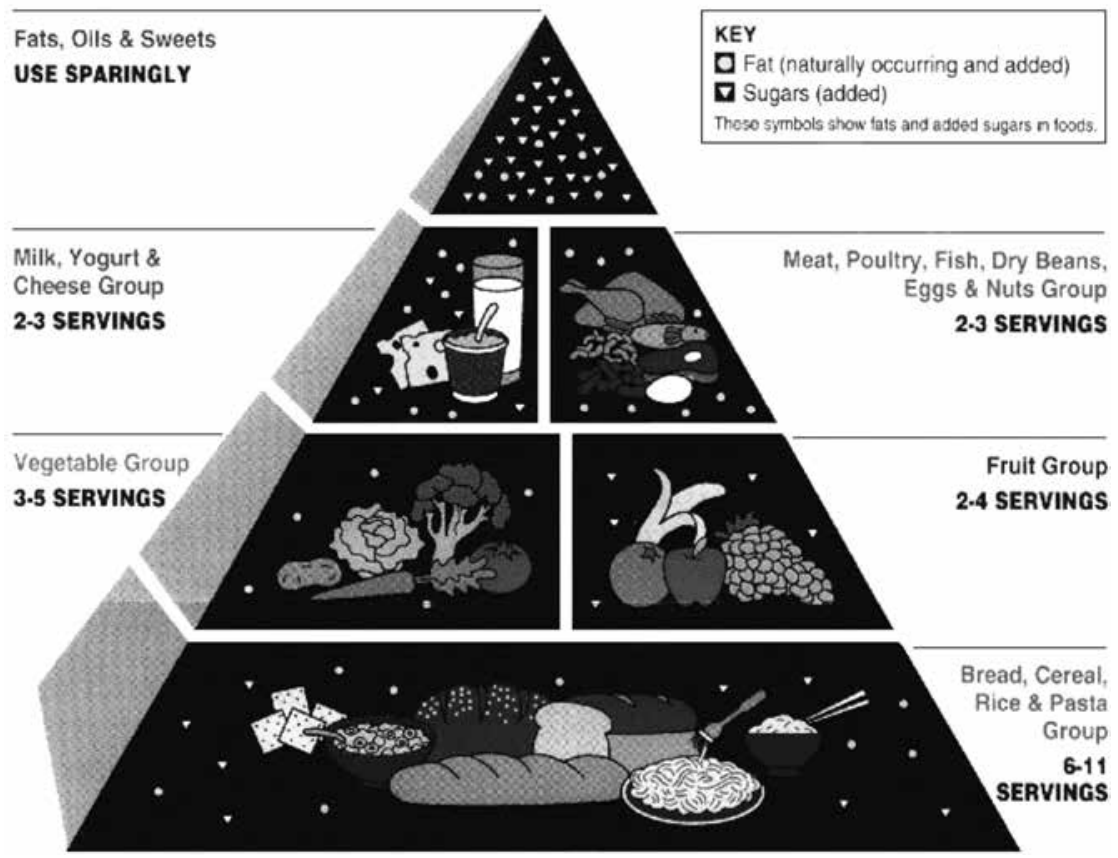

USDA (s. f.). Food Guide Pyramid. Recuperada de http://www.cnpp.usda.gov/FGP 
2. My Healthy Eating Plate (Libre de usarse para propósitos no comerciales)

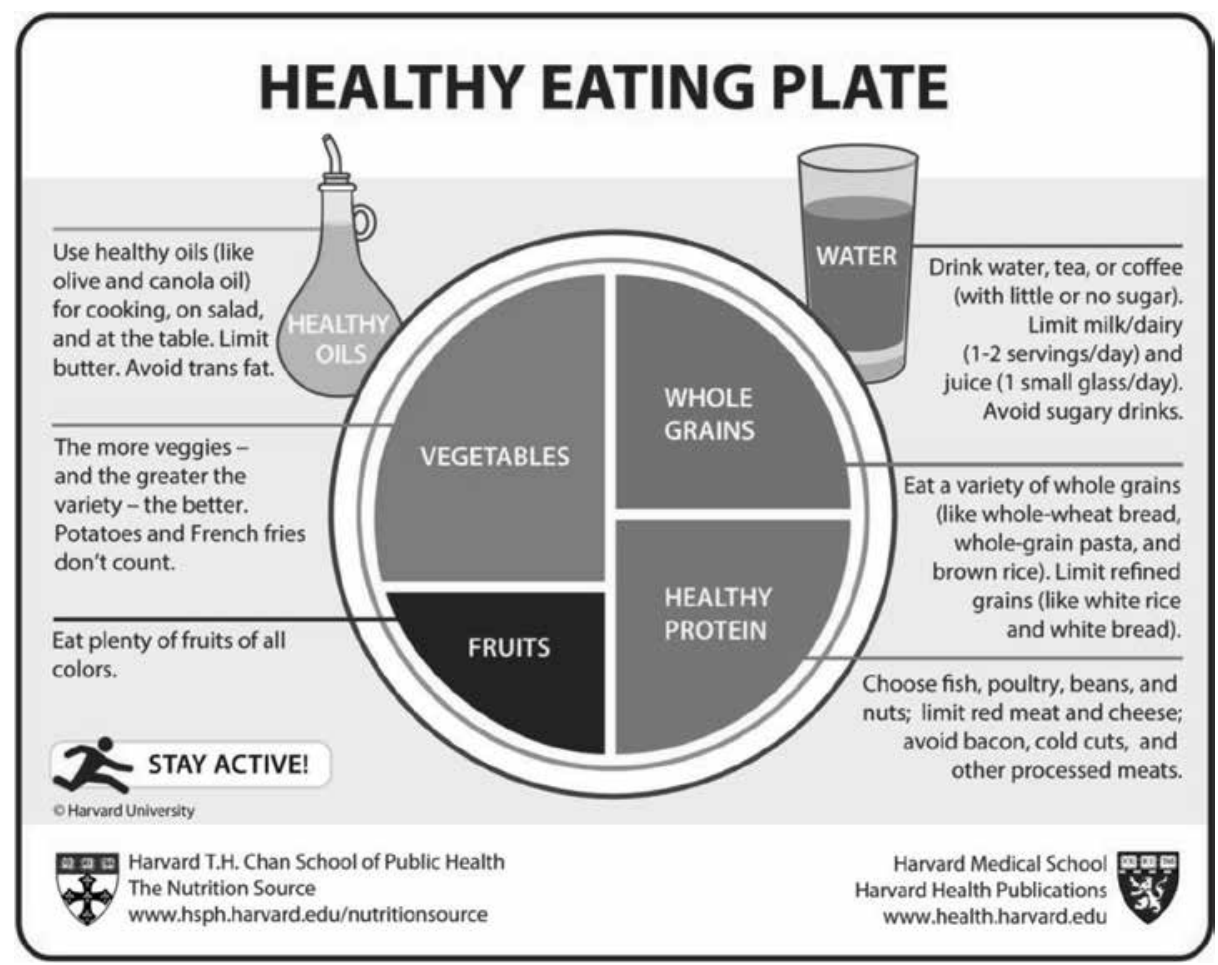

Copyright (C) 2011, Harvard University. For more information about The Healthy Eating Plate, please see The Nutrition Source, Department of Nutrition, Harvard School of Public Health, www.thenutritionsource.org, and Harvard Health Publications, www.health.harvard.e 
3. Rate Your Own Pyramid (Material fotocopiado, recolectado en Saint Michael's College, Vermont).

\section{Rate Your Own Pyramid}

Use the following food pyramids to see how you personally are doing in achieving a well balanced diet. Use the back of this page for a reference as to serving sizes for each food group.

Day 1

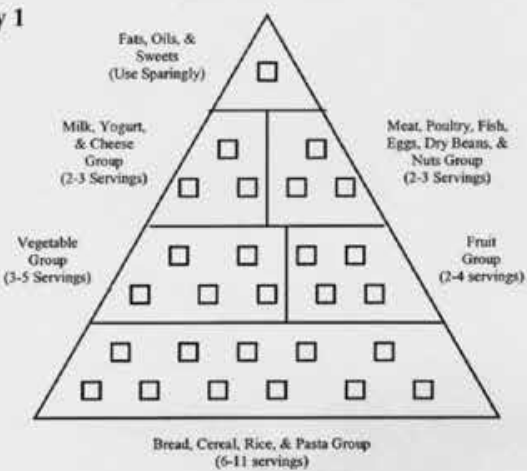

Tay 3

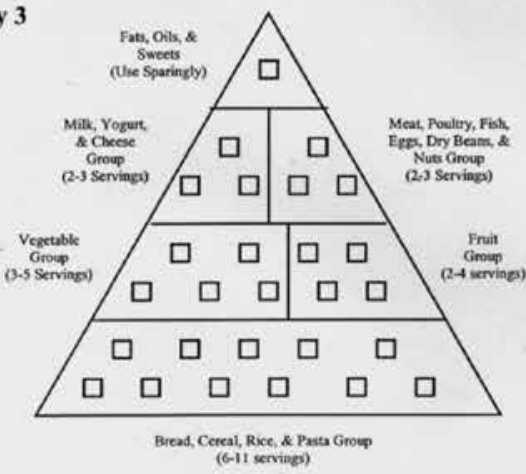

Day 2

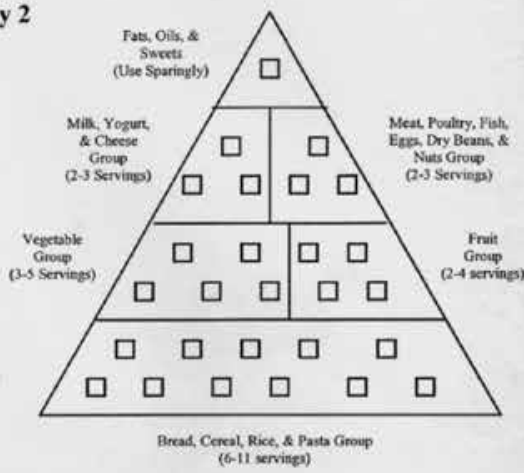

Day 4

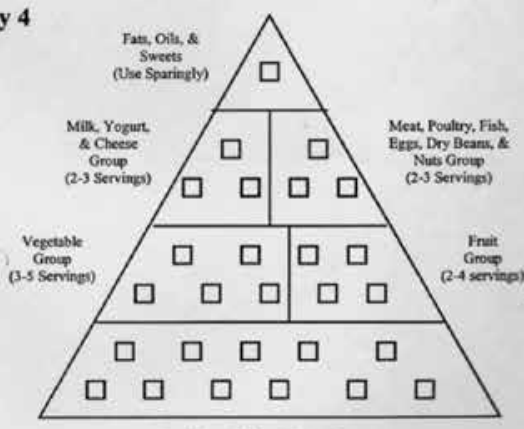

Bread, Cereal, Rice, \& Pasta Group

Did your pyramids improve by day four? Sometimes if we pay a little more attention to the big picture and what we are getting over the course of a couple days, we can see what food groups we are lacking. 
4. 24 Hour Recall (Diseñado con base en Mataix Verdú. J, García Diz, L., Llopis González, J., s.f y Carbajal Azcona, Á. 2004)

\section{Hour Recall}

Dear participant, thanks for taking your time to complete this survey. Provide the personal information requested. Then, do you best to remember what you ate during the last 24 hours. Check all the ones that apply and use the spaces to list any additional foods that you had. Provide the time, place, and the ingredients added. Estimate how much of each food you had. Thanks for your help.
Gender:
Height:
BMI:
Age:
Weight:

Breakfast

\begin{tabular}{|l|l|l|l|l|l||}
\hline Food & \multicolumn{3}{|c|}{ Time } & \multicolumn{3}{c|}{ Home } & \multicolumn{2}{c|}{ Grl or } \\
Bread & & & & & \\
\hline Cookies & & & & & \\
\hline Tortillas & & & & & \\
\hline Gallo pinto & & & & & \\
\hline Cheese & & & & & \\
\hline Sour cream & & & & & \\
\hline Coffee & & & & & \\
\hline Juice & & & & & \\
\hline Fruit & & & & & \\
\hline & & & & & \\
\hline & & & & & \\
\hline & & & & & \\
\hline
\end{tabular}


Lunch

\begin{tabular}{|c|c|c|c|c|c|}
\hline Food & Time & Place & Ingredients & $\begin{array}{l}\text { Home } \\
\text { Measure }\end{array}$ & $\begin{array}{l}\text { Grl or } \\
\mathrm{ml}\end{array}$ \\
\hline \multicolumn{6}{|l|}{ Rice } \\
\hline \multicolumn{6}{|l|}{ Beans } \\
\hline \multicolumn{6}{|l|}{ Pasta } \\
\hline \multicolumn{6}{|l|}{ Salad } \\
\hline \multicolumn{6}{|l|}{ Meat } \\
\hline \multicolumn{6}{|l|}{ Chicken } \\
\hline \multicolumn{6}{|l|}{ Fish } \\
\hline \multicolumn{6}{|l|}{ Vegetables } \\
\hline \multicolumn{6}{|l|}{ Cold meats } \\
\hline & & & & & \\
\hline & & & & & \\
\hline & & & & & \\
\hline
\end{tabular}

Dinner

\begin{tabular}{|c|c|c|c|c|c|}
\hline Food & Time & Place & Ingredients & $\begin{array}{l}\text { Home } \\
\text { Measure }\end{array}$ & $\begin{array}{l}\text { Grl or } \\
\mathrm{ml}\end{array}$ \\
\hline \multicolumn{6}{|l|}{ Rice } \\
\hline \multicolumn{6}{|l|}{ Beans } \\
\hline \multicolumn{6}{|l|}{ Pasta } \\
\hline \multicolumn{6}{|l|}{ Salad } \\
\hline \multicolumn{6}{|l|}{ Meat } \\
\hline \multicolumn{6}{|l|}{ Chicken } \\
\hline \multicolumn{6}{|l|}{ Fish } \\
\hline \multicolumn{6}{|l|}{ Cold meats } \\
\hline & & & & & \\
\hline & & & & & \\
\hline & & & & & \\
\hline
\end{tabular}


Snack

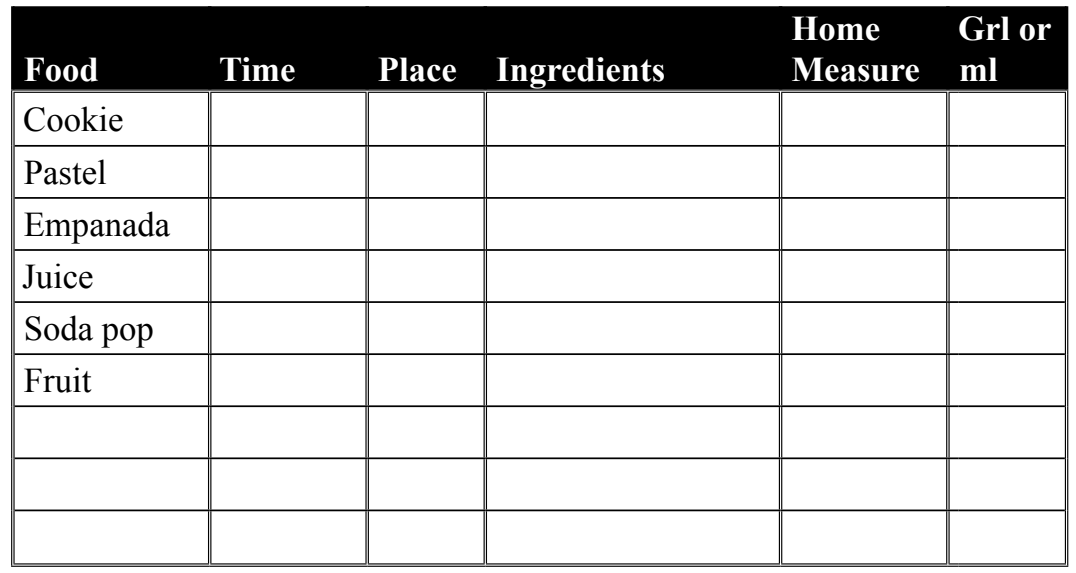

Nota: Elaboración propia, 2016. 